\title{
Subtenon Triamcinolone Acetonide Injection with Topical Anesthesia in Pediatric Non-Infectious Uveitis
}

Jennifer L. Jung • Mariana Harasawa · Jennifer L. Patnaik •

Alan G. Palestine

Received: December 31, 2021 / Accepted: February 3, 2022 / Published online: February 18, 2022

(C) The Author(s) 2022

\section{ABSTRACT}

Introduction: Subtenon triamcinolone acetonide (Kenalog ${ }^{\circledR}$; Bristol Myers Squibb) (STA) injections are commonly used in the treatment of adults in an outpatient setting. However, publications on detailing its outpatient use, safety, and efficacy in the pediatric population are scarce.

Methods: We reviewed STA injections performed in children in the outpatient clinics at two tertiary centers from 2014 to 2020. All children were aged $\leq 18$ years and had a diagnosis of non-infectious uveitis. STA injections were done using $0.5 \mathrm{cc}(20 \mathrm{mg})$ triamcinolone injected superotemporally with only topical anesthesia. Data on the efficacy and safety of STA in treating inflammation and compiled data on visual acuity improvement and inci-

J. L. Jung · J. L. Patnaik · A. G. Palestine $(\bowtie)$ Department of Ophthalmology, University of Colorado School of Medicine, 1675 Aurora Court, Mail Stop F731, Aurora, CO 80045, USA

e-mail: alan.palestine@cuanschutz.edu

M. Harasawa

Omni Eye Specialists, EyeCare Services Partner, Denver, CO 55 Madison Street, Suite 355, Denver, CO 80206, USA dence of ocular complications were evaluated.

Results: Forty-eight eyes in 30 patients were included. The mean age of patients was 13.1 (range 7-18) years. There were no immediate complications observed in all injections performed. At the 3-month follow-up, inflammation had improved in $85.4 \%$ of eyes, macular edema had resolved in $77.8 \%$ of eyes, and there was significant vision improvement after STA. At 6 months after STA, the incidence of ocular hypertension was $12.5 \%$ and no new cataracts had developed.

Conclusion: STA injection with topical anesthesia was a well-tolerated, reasonable alternative for short-term treatment of uveitis among this pediatric population.

Keywords: Macular edema; Ocular hypertension; Pediatric non-infectious uveitis; Pediatric uveitis; Periocular corticosteroid injection; Subtenon triamcinolone acetate injection; Topical anesthesia; Uveitic cataract 


\section{Key Summary Points}

Why carry out this study?

Pediatric uveitis has a high rate of ocular complications that can result in permanent vision loss.

Subtenon triamcinolone acetate (STA) injections are commonly used in the outpatient clinic setting to treat uveitis and macular edema in adults, but the outpatient use in pediatric patients has not been reported.

We hypothesized that STA with topical anesthesia alone can be a safe and effective treatment option in pediatric uveitis.

\section{What was learned from the study?}

Treatment with STA with topical anesthesia resulted in improvement of inflammation for $85.4 \%$ of eyes and resolution of macular edema in $77.8 \%$ at 3 months post treatment.

At 6 months after STA, incidence of ocular hypertension was $12.5 \%$ and no new cataracts developed.

STA with topical anesthesia can be a useful option for short-term management of pediatric uveitis.

\section{INTRODUCTION}

Pediatric uveitis is uncommon when compared to uveitis in the adult population, with the former accounting for between 2.2 to $13.8 \%$ of patients in many uveitis clinics $[1,2]$. However, prognosis of uveitis can be worse in children due to high rates of sight-threatening complications during amblyogenic age as well as challenges surrounding treatment and its side effects [2, 3].
The standard first-line treatment for non-infectious uveitis is corticosteroids. If topical corticosteroids have failed, oral corticosteroids are a standard short-term option. However, these can be associated with weight gain, acne, mood changes, metabolic issues, and growth retardation. Furthermore, oral corticosteroids may need to be continued for 3-12 months as longterm systemic immunosuppression is begun and an effective regimen is established. Periocular triamcinolone acetonide (PTA) injection can be performed as a localized deposition of corticosteroid into the orbital floor or as subtenon triamcinolone acentonide (STA) injection. PTA provides more posterior antiinflammatory effect and a more consistent drug concentration compared to topical therapy, while minimizing systemic absorption $[4,5]$.

PTA injections are frequently used in adults to treat uveitis and macular edema in the outpatient setting [5-8]. However, there is scarcity of published reports on the use of PTA in pediatric uveitis. In 1977, Giles suggested that PTA should be considered as an alternative to oral corticosteroids while awaiting systemic immune suppression to take full effect [9]. In 2010, Habot-Wilner et al. reported 15 consecutive children who received PTA under general anesthesia and had significant improvement of inflammation [10]. Both authors recommended general anesthesia for PTA injection in children aged $<16$ years. The need for general anesthesia and concerns regarding the development of ocular complications associated with PTA have led to hesitation for its use in children.

In this study, we report the use of STA for uveitis in a pediatric population in an outpatient setting with topical anesthesia alone. The primary objectives were to evaluate the effectiveness of STA in treating inflammation and safety with only topical anesthesia in an outpatient setting. Secondary objectives were the assessment of visual acuity and incidence of potential ocular complications, including ocular hypertension and cataract formation. 


\section{METHODS}

We conducted a retrospective chart review on all consecutive children with active non-infectious uveitis who underwent STA injections in the outpatient clinics at two tertiary centers from 2014 to 2020. Data collection included dates of injections, laterality, classification of uveitis, best-corrected Snellen visual acuity, inflammation level before and after the first injection, results of ancillary imaging, medications, and any procedure-related or ocular complications. This study was approved by the Colorado Multiple Institution Review Board and conformed to the requirements of the United States Health Insurance Portability and Privacy Act. This study conformed with the Helsinki Declaration of 1964, as revised in 2013, concerning human and animal rights, and Springer's policy concerning informed consent was followed.

The inclusion criteria were age $\leq 18$ years, diagnosis of non-infectious uveitis, and followup visit within 3 months after the first injection. This time frame was chosen as the effect of STA peaks 2-3 months after injection [8].

Inflammation was classified based on the Standardization of Uveitis Nomenclature (SUN) Working Group criteria [11]. We used anterior chamber cell grading for patients with diagnosis of anterior uveitis and anterior vitreous cell grading for patients diagnosed with intermediate and posterior uveitis. Active inflammation was defined by one or more of the following: (1) presence of anterior chamber (AC) cells $>0.5+$ cells according to the SUN criteria; (2) vitreous cells $>0.5+$ cells; or (3) presence of macular edema (ME) and/or optic nerve edema (ONE). Since there is no agreed-upon method for vitreous cell grading and because many patients do not have significant vitreous haze, we adopted a grading system for vitreous cells that parallels the SUN criteria for AC cells by examining the anterior vitreous on slit-lamp examination, as previously used in other studies $[6,10]$. ME was defined as petalloid leakage on fluorescein angiogram (FA) or central macular thickness $\geq 300 \mu \mathrm{m}$ or presence of cystic intraretinal fluid on optical coherence tomography (OCT;
Heidelberg Spectralis; Heidelberg, Germany). Thickness of $\geq 300 \mu \mathrm{m}$ was chosen based on the normative reference of $274 \mu \mathrm{m}$ determined by Yanni et al. for the age group of 5-15 years [12]. The presence of ONE was determined by examation. Success was defined as improvement of inflammation at 3 months after STA injection. Improvement in inflammation was defined as a two-step decrease in the level of inflammation or a decrease to inactive level.

Ocular hypertension (OHT) was defined as rise in $>10 \mathrm{mmHg}$ points in intraocular pressure (IOP) from baseline at two consecutive followup visits or an IOP of $>30 \mathrm{mmHg}$ on a single follow-up visit, with or without glaucomatous change observed in the optic nerve. Since the effect of STA can peak at around 3 months and fade at around 6 months, IOP data for the period up to 6 months after STA injection were used to determine the incidence of OHT. Cataract was defined as any lens opacity seen on slit-lamp examination. Presence of cataracts was recorded for the duration of the follow-up for each patient and incidence analyzed at 6 months and 1 and 2 years after STA. Bestcorrected vision was measured in Snellen visual acuity and converted to logarithm angle of resolution (logMAR) prior to STA treatment and at the 3-month follow-up visit.

\section{Patient Selection}

Selection of a suitable candidate for this procedure is critical during the pre-procedure examination to ensure patient safety and cooperation. Suitable candidates were patients who met the inclusion criteria and had calm, cooperative demeanors and a good interaction with the provider. STA was not offered to anxious, fearful patients with dependent attitude towards the parents, and those who did not respond well to verbal instructions. The procedure was discussed first with the parents alone and then with the patients, taking into account the age of the patient. 


\section{Injection Technique}

Informed consent was obtained from parents prior to the injection, and the appropriate eye was marked. The procedure consists of first placing multiple proparacaine drops on the eye, following which the child is instructed to look infero-nasally, thereby exposing the superotemporal quadrant and keeping the needle out of the patient's view. Then, $0.5 \mathrm{ml}(20 \mathrm{mg})$ of triamcinolone acetonide $40 \mathrm{mg} / \mathrm{ml}$ (Kenalog ${ }^{\circledR}$; Bristol-Meyers Squibb, New York, NY, USA) is injected using a half-inch 27-gauge needle into the superotemporal subtenon's space. The medication is injected as posteriorly as possible, while still maintaining visualization of the needle tip, creating a visible corticosteroid bleb. The final position of the steroid depot lies approximately $15 \mathrm{~mm}$ posterior to the limbus. Injection should be done rapidly over a few seconds as a slow injection leads to increased anxiety in the patient. All STA injections were performed by two of the authors (JLJ and AGP).

While a volume of $1 \mathrm{ml}$ (40 mg) (Kenalog) is commonly used as STA in adults, a smaller volume $(0.5 \mathrm{ml})$ was chosen for our pediatric patients because (1) children have smaller orbits and (2) a small volume allows for more rapid injection and a lower sensation of pressure under topical anesthesia.

\section{Statistical Analysis}

Descriptive statistics included basic frequencies for categorical variables and means, medians and ranges for continuous variables at the patient and ocular levels. A Kaplan-Meier failure curve was plotted for time to first cataract development for eyes, excluding those who had cataracts prior to STA. Pre-operative and postoperative visual acuity were plotted for both right and left eyes. The signed-rank test for nonparametric data was used for comparing vision before and after STA.
Table 1 Patient- and eye-specific characteristics at the time of the first subtenon triamcinolone acetate injection

\begin{tabular}{|c|c|}
\hline Characteristics & $N(\%)$ \\
\hline Unilateral uveitis & $12(40 \%)$ \\
\hline Male patients & $15(50 \%)$ \\
\hline \multicolumn{2}{|l|}{ Diagnosis } \\
\hline Pars planitis & $19(63 \%)$ \\
\hline Chronic anterior uveitis & $4(13 \%)$ \\
\hline Panuveitis & $3(10 \%)$ \\
\hline $\begin{array}{l}\text { HLA-B27 associated recurrent } \\
\text { uveitis }\end{array}$ & $3(10 \%)$ \\
\hline Vogt-Koyanagi-Harada & $1(3 \%)$ \\
\hline Presence of $\mathrm{ME}$ & $18 / 48$ eyes $(37.5 \%)$ \\
\hline Presence of ONE & $9 / 48$ eyes $(18.8 \%)$ \\
\hline Presence of cataracts & $9 / 48$ eyes $(18.8 \%)$ \\
\hline \multicolumn{2}{|l|}{ Medications prior to STA } \\
\hline Topical corticosteroids & $28(93.3 \%)$ \\
\hline Oral corticosteroids & $10(33.3 \%)$ \\
\hline Systemic immunosuppressants & $7(23.3 \%)$ \\
\hline IOP-lowering medication & $2(6.7 \%)$ \\
\hline Mean age at first STA injection & $\begin{array}{l}13.1 \text { years (range } \\
7-18)\end{array}$ \\
\hline $\begin{array}{l}\text { Patients that received } \geq 1 \text { STA } \\
\text { injections }\end{array}$ & $15(50 \%)$ \\
\hline
\end{tabular}

$M E$ Macular edema, ONE optic nerve edema, IOP intraocular pressure, $S T A$ subtenon triamcinolone acetonide

\section{RESULTS}

A total of 34 patients (54 eyes) received STA injections in the outpatient clinic setting, of whom 30 patients (48 eyes) were included in the study. Four patients were excluded due to lack of follow-up data within a 3-month period for primary outcome assessment, but all tolerated the procedure well with no immediate complications. Table 1 shows the patient and ocular characteristics. 
Table 2 Primary and secondary outcomes of the study for eyes after subtenon triamcinolone acetonide injection

\begin{tabular}{ll}
\hline Outcomes & $N(\%)$ \\
\hline Success at 3 months after STA & $41 / 48(85.4 \%)$ \\
Resolution of ME & $14 / 18(77.8 \%)$ \\
Resolution of ONE & $9 / 9(100 \%)$ \\
New cataract formation ${ }^{\mathrm{a}}$ & \\
At 6 months after STA & 0 \\
At 1 year after STA & $9 / 39(23.7 \%)$ \\
At 2 years after STA & $17 / 39(43.6 \%)$ \\
Ocular hypertension incidence & $6 / 48(12.5 \%)$ \\
At 6 months & \\
\hline $\begin{array}{l}\text { ancludes eyes that received intravitreal medications after } \\
\text { STA }\end{array}$
\end{tabular}

Indications for STA injection included one of more of the following: persistent inflammation on topical corticosteroids $(n=28)$; wish to stop systemic corticosteroids $(n=10)$; unable to start on corticosteroids or immunomodulating treatment (IMT) due to other systemic concerns $(n=2)$; and need for short-term control while bridging to new systemic IMT $(n=11)$. There were no immediate procedure-related complications observed in all injections performed. The average duration of follow-up after STA injection was 35.3 months (median 32 months, range 1-78 months). Table 2 summarizes the primary and secondary outcomes.

\section{Inflammation Results}

Success was achieved in 41 of 48 eyes $(85.4 \%)$ at the endpoint of 3 months after STA. Seven eyes did not show improvement in inflammation by our definition of either an improvement by 2 grades or "inactivity," although all showed a 1 grade improvement in inflammation and improvement seen on ancillary testing (less leakage on FA and decreased macular thickness on OCT). No eyes had a worsening of inflammation after STA.
Eighteen eyes (37.5\%) had ME on FA or OCT prior to STA, of which 14 (77.8\%) had resolution of ME within 3 months of STA. Three eyes of the remaining four eyes showed improvement upon examination and ancillary testing, but with persistent ME according to study definition (CMT $\geq 300 \mu \mathrm{m}$ ), and the remaining eye had no improvement. Of the 48 eyes, ten $(20.8 \%)$ had ONE prior to STA, and all had improvement of edema at 3 months after STA.

After the initial resolution of uveitis following STA, four (9.1\%) of 44 eyes had recurrence within 1 year after STA (median 4 months). A second STA injection was given to two eyes after which uveitis remained controlled for $>1$ year. One patient, who had recurrence in both eyes 4 months after STA, achieved control of uveitis with additional treatment with topical corticosteroids and IMT.

Before receiving STA, 28 of 30 patients were on topical corticosteroids, ten patients were on oral corticosteroids (prednisone: median dose $17.5 \mathrm{mg}$, range $4-40 \mathrm{mg}$ ), and seven patients were on systemic IMT. Corticosteroids were tapered and stopped in all 30 patients after STA. At the final follow-up, 15 patients (50\%) were on IMT. Twelve patients did not require longterm IMT as inflammation was controlled after STA (average follow-up 34 months, median 28 months, range $1-63$ months). Of these 12 patients (20 eyes), seven (12 eyes) had quiescence after one STA injection and five patients (8 eyes) had quiescence after two STA injections. One patient with unilateral disease who experienced multiple side effects with various types of IMT was able to stop all systemic medications after one STA injection.

\section{Cataract Results}

Twenty-three patients (39 eyes) did not have cataracts prior to STA injections. The incidence of new cataract was zero at 6 months after STA. New cataracts were seen in six patients ( 9 eyes) at 1 year after STA injection, and in an additional five patients (eight eyes) at 2 years after STA injection (Table 2). Three of these patients had intravitreal injections, which is more likely to have contributed to cataract development. 


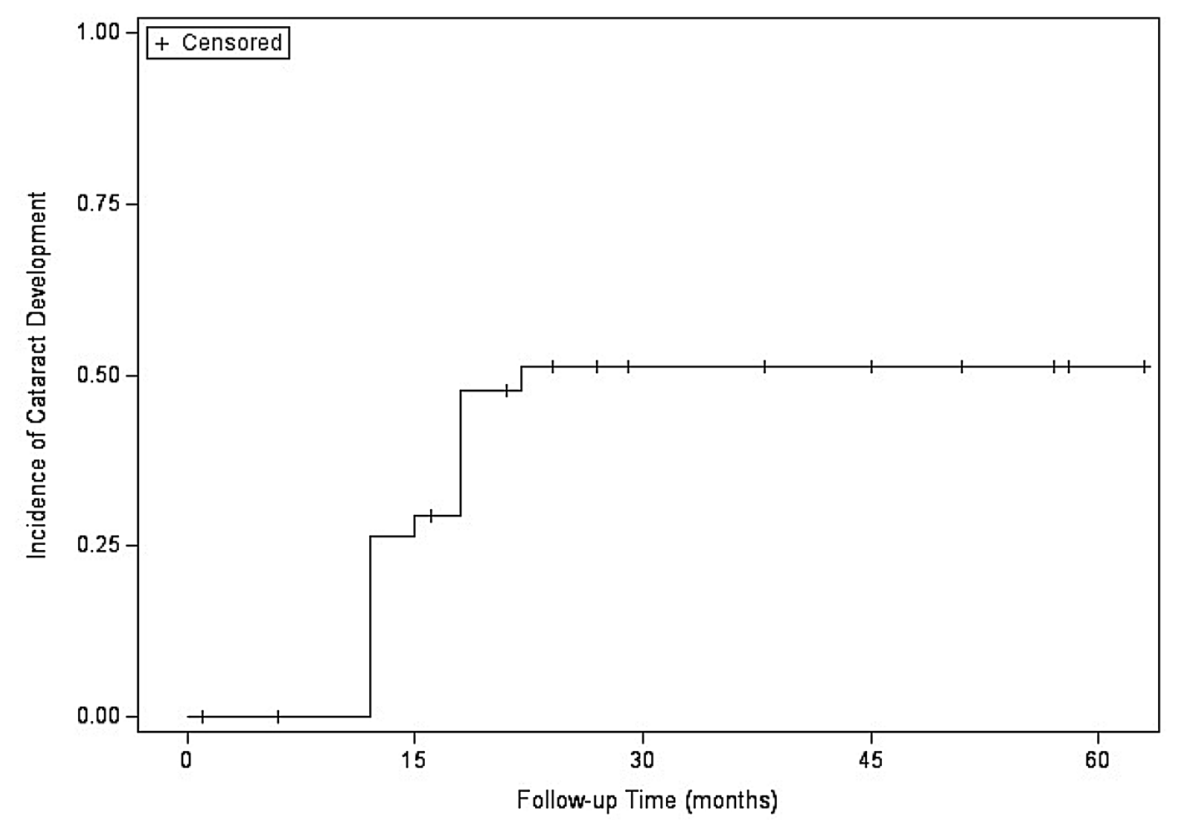

Fig. 1 Kaplan-Meier curve showing the cumulative incidence of cataract development over time after subtenon triamcinolone acetonide injection

Two patients received intravitreal dexamethasone implant (Ozurdex ${ }^{\circledR}$; Allergan Inc., Dublin, Ireland) at 7 and 9 months after the respective STA injection, and one patient received intravitreal methotrexate 3 months after STA injection. Figure 1 shows the Kaplan-Meier curve for the development of cataracts after STA, including the patients who received intravitreal injections after STA. Cataracts occurred between 12 and 22 months following STA treatment and remained stable after that point.

Excluding the three patients with other significant cataract-inducing factors in addition to STA, five of 33 eyes (15\%) and 11 of 33 eyes (33.3\%) developed cataracts of varying severity associated with STA by 1 year and 2 years after STA, respectively. Of the 11 eyes that developed cataract by 2 years after STA, six received more than 1 STA injection. Cataract surgery was performed for three of the 11 eyes within 3 years after STA. The remaining eyes maintained good visual acuity of $20 / 25$ or better at the last followup (average 31.7 months, median 27 months, range $12-57$ months).
Twenty-two eyes did not develop any lens opacities at the last follow-up (average 28.8 months, median 28 months, range 1-63 months), of which eight eyes received $>1$ injection.

\section{Ocular Hypertension Results}

Of the total 48 eyes, six (12.5\%) developed OHT within 6 months after STA, with all six eyes (four patients) requiring IOP-lowering medications. One additional patient was started on an IOP-lowering drop due to a 7-point rise in IOP. Ocular hypertension developed in four of these six eyes within 4 weeks of STA. IOP was controlled with one IOP-lowering drop in five of the six eyes. Three eyes eventually received glaucoma surgery: one eye for persistently elevated IOP, and two eyes in one patient whose IOP was controlled on topical treatment underwent combined cataract and glaucoma surgery 1 year after STA. 


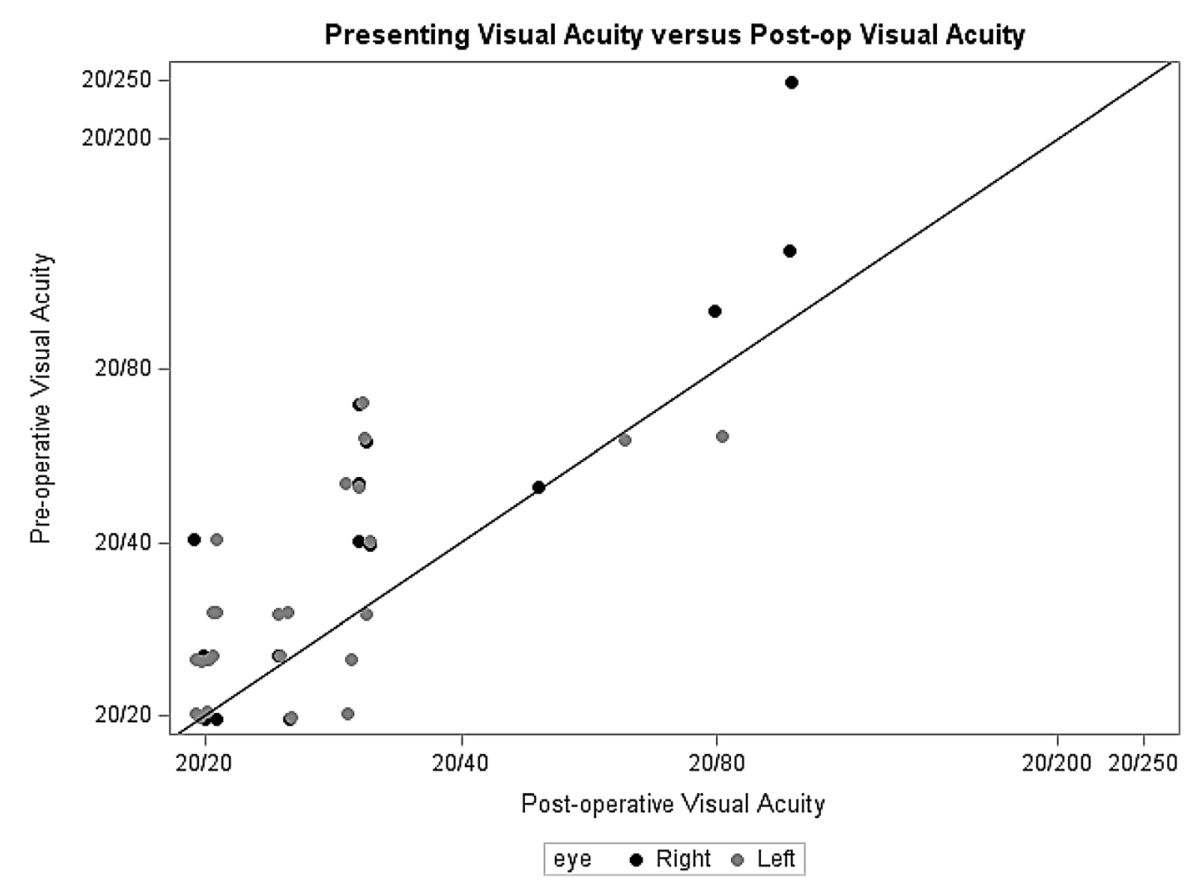

Fig. 2 Visual acuity before and after subtenon triamcinolone acetonide injection

\section{Vision Results}

There was significant improvement in vision after STA in both eyes: right eyes $(n=19$, $p=0.0002)$ and left eyes $(n=25, p=0.007)$ (Fig. 2).

\section{DISCUSSION}

Our study demonstrated that STA injection with topical anesthesia in the outpatient setting is a feasible, effective treatment option for children with uveitis. In previously published studies of PTA in uveitis, inflammation control was achieved in $48-74 \%$ of eyes and resolution of ME in $45-86 \%$ of eyes $[6,7,10,13]$. In our study, the presence of $0.5+$ cells or fewer was considered to indicate inactivity, similar to the criteria of inactivity used in the Childhood Arthritis and Rheumatology Research Alliance Consensus guideline for chronic anterior uveitis [14]. This criterion was chosen because in a practical setting, escalation of treatment is not pursued based on the presence of $0.5+$ cells. If we did not include patients with $0.5+$ cells in the success group, our success rate decreases to $75 \%$, which is comparable to that of the Systemic Immunosuppressive Therapy for Eye Diseases (SITE) cohort [7]. In contrast, the study by Salek et al. used similar AC/vitreous grading for inflammation to our study and showed that in 81 patients (109 eyes) with uveitis, $48 \%$ of eyes achieved inflammation control and $45 \%$ of eyes had resolution of macular edema at 3 months after PTA. The percentages of patients on oral corticosteroids and IMT are comparable to our study.

While the above two studies included both adults and children, Habot-Wilner et al. reported a series of 15 children (19 eyes) who underwent orbital floor PTA under general anesthesia. Inflammation improved in 14 of 19 eyes $(74 \%)$ by a median of 4 weeks, and ME resolved in six of 11 eyes (55\%) after PTA [10]. However, despite the high rate of initial control of inflammation, the relapse rate was much higher than that in our study (50 vs. $10 \%$, respectively), possibly related to our successful initiation of IMT after STA. The indication for 
PTA in ten of the 19 eyes in the Habot-Wilner study was to avoid escalating IMT, which differs from the goal of our study.

Compared to adults, children with pediatric uveitis experience higher rates of ocular complications. Rosenberg et al. evaluated a cohort of 148 children with uveitis and reported that $34 \%$ had at least one ocular complication at the time of diagnosis, $75.2 \%$ by 1 year, and $86.3 \%$ by 3 years [2]. In our study, the most common complication present prior to STA was macular edema (37.5\%), followed by uveitic cataract (18.8\%). Retrospective studies in pediatric uveitis report cataract prevalence ranging from $42.8 \%$ to $69.9 \%[2,3,15,16]$. Prior studies specifically addressing the use of PTA in uveitis evaluated cataract incidence in shorter time frames in order to assess a more direct association with injections and reported $36.3 \%$ of new cataracts by 6 months and 20.2-28.5\% by 12 months $[6,7,10]$. In our cohort, no eyes developed new cataracts at 6 months after STA and $23.7 \%$ developed cataracts at 12 months after the procedure.

The prevalence of OHT/secondary glaucoma in pediatric uveitis is reported as ranging from $26.3 \%$ to $50.7 \%[2,3,15,17]$. The incidence of OHT after PTA ranges from $16.3 \%$ to $22 \%$ $[6,7,18]$. Kothari et al. evaluated 916 children with uveitis (1593 eyes) and defined OHT in a similar manner as in our study. A dose-response relationship of corticosteroids and OHT was reported where PTA had a seven- to eightfold hazard risk, which was still comparable to the risk of cataract when using $>4$ drops of topical prednisolone acetate daily [19]. In our cohort, OHT occurred in $12.5 \%$ of eyes, which may be due to the lower dose of triamcinolone. Our study is the first to demonstrate the efficacy of a reduced dose of STA for pediatric uveitis.

Not all children are good candidates for STA injection with topical anesthesia. In our clinics, we found that the application of patient selection criterion to screen for suitable patients was a crucial step in identifying suitable patients to ensure patient safety. In our experience, the best approach is to reserve injections until after the initial visit in order to build good rapport with the patient and the parents. Although the exact behavioral characteristics indicating suitability are a subjective assessment, we based this decision on the level of cooperation of the child during the examination and the ability of the child to follow instructions.

Our study has several limitations, including the retrospective nature of the study. Patient selection for STA injection was at the discretion of the treating physician, which therefore induced selection bias. Another limitation is the lack of consensus in vitreous cell grading. During the development of the Standardized Uveitis Nomenclature in 2005, no consensus was reached for vitreous cell grading [11]. Vitreous haze is not present in all types of uveitis, and although the National Eye Institute system of vitreous haze can be used, it is not an ordinal system, and levels represent a non-linear hierarchy of increasing magnitude without a numerical relationship to the amount of inflammation. However, we used criteria similar to other studies $[6,10]$ and maintained consistency across the two treating physicians.

\section{CONCLUSIONS}

In conclusion, our results show STA with topical anesthesia in the pediatric population is a welltolerated, safe treatment option for pediatric uveitis. It is highly effective in improving inflammation, vision, and macular edema. It aided in the reduction of corticosteroid use and in effective transition to systemic IMT. The reduced dose of $20 \mathrm{mg}$ triamcinolone may decrease the risk of OHT compared to the standard dose of $40 \mathrm{mg}$, although it is associated with a similar risk of cataract formation. The treatment regimen should be individualized based on the patient's needs, severity of inflammation, and presence of ocular complications. Thus, STA with topical anesthesia can provide one more option in our toolbox for treating this complicated disease in children.

\section{ACKNOWLEDGEMENTS}

We express our deep appreciation to the participants of this study. 
Funding. This study was supported in part by an unrestricted grant to the Department of Ophthalmology, University of Colorado from Research to Prevent Blindness Inc, New York, NY, which had no influence on the intellectual content, and by the Center for Ocular Inflammation, Department of Ophthalmology, University of Colorado. No funding or sponsorship was received for publication of this article.

Authorship. All named authors meet the International Committee of Medical Journal Editors (ICMJE) criteria for authorship for this article, take responsibility for the integrity of the work as a whole, and have given their approval for this version to be published.

Author Contributions. JJ contributed to: concept and design, data gathering and analysis, drafting, and editing the manuscript. $\mathrm{MH}$ contributed to: data gathering and drafting the manuscript. JP contributed to: statistical analysis and editing the manuscript. AP contributed to: concept and design, statistical analysis, and editing the manuscript.

Disclosures. Jennifer Jung, Mariana Harasawa, Jennifer Patnaik, and Alan Palestine declare that they have no conflict of interest.

Compliance with Ethics Guidelines. This study was approved by the Colorado Multiple Institution Review Board and conformed to the requirements of the United States Health Insurance Portability and Privacy Act. This study conformed with the Helsinki Declaration of 1964, as revised in 2013, concerning human and animal rights, and Springer's policy concerning informed consent was followed.

Data Availability. The datasets generated during and/or analyzed during the current study are available from the corresponding author on reasonable request.

Open Access. This article is licensed under a Creative Commons Attribution-NonCommercial 4.0 International License, which permits any non-commercial use, sharing, adaptation, distribution and reproduction in any medium or format, as long as you give appropriate credit to the original author(s) and the source, provide a link to the Creative Commons licence, and indicate if changes were made. The images or other third party material in this article are included in the article's Creative Commons licence, unless indicated otherwise in a credit line to the material. If material is not included in the article's Creative Commons licence and your intended use is not permitted by statutory regulation or exceeds the permitted use, you will need to obtain permission directly from the copyright holder. To view a copy of this licence, visit http://creativecommons.org/licenses/by$\mathrm{nc} / 4.0 /$.

\section{REFERENCES}

1. Smith JA, Mackensen F, Sen HN, et al. Epidemiology and course of disease in childhood uveitis. Ophthalmology. 2009;116(8):1544-51.

2. Rosenberg KD, Feuer WJ, Davis JL. Ocular complications of pediatric uveitis. Ophthalmology. 2004;111(12):2299-306.

3. Kump LI, Cervantes-Castaneda RA, Androudi SN, Foster CS. Analysis of pediatric uveitis cases at a tertiary referral center. Ophthalmology. 2005;112(7):1287-92.

4. McKay KM, Borkar DS, Sevgi DD, Susarla G, Papaliodis GN, Sobrin L. Comparison of modified posterior sub-tenon's vs. trans-septal triamcinolone injection for non-infectious uveitis. Ocul Immunol Inflamm. 2020;2:1-8.

5. Thomas AS, Lin P. Local treatment of infectious and noninfectious intermediate, posterior, and panuveitis: current concepts and emerging therapeutics. Curr Opin Ophthalmol. 2020;31(3):174-84.

6. Salek SS, Leder HA, Butler NJ, Gan TJ, Dunn JP, Thorne JE. Periocular triamcinolone acetonide injections for control of intraocular inflammation associated with uveitis. Ocul Immunol Inflamm. 2013;21(4):257-63.

7. Sen HN, Vitale S, Gangaputra SS, et al. Periocular corticosteroid injections in uveitis: effects and complications. Ophthalmology. 2014;121(11): 2275-86. 
8. Thorne JE, Sugar EA, Holbrook JT, et al. Periocular triamcinolone vs intravitreal triamcinolone vs intravitreal dexamethasone implant for the treatment of uveitic macular edema: The PeriOcular vs INTravitreal corticosteroids for uveitic macular edema (POINT) trial. Ophthalmology. 2019;126(2): 283-95.

9. Giles CL. Peripheral uveitis in children. J Pediatr Ophthalmol. 1977;14(2):93-5.

10. Habot-Wilner Z, Sallam A, Roufas A, et al. Periocular corticosteroid injection in the management of uveitis in children. Acta Ophthalmol. 2010;88(8): e299-304.

11. Jabs DA, Nussenblatt RB, Rosenbaum JT, Standardization of Uveitis Nomenclature Working G. Standardization of uveitis nomenclature for reporting clinical data. Results of the First International Workshop. Am J Ophthalmol. 2005;140(3):509-16.

12. Yanni SE, Wang J, Cheng CS, et al. Normative reference ranges for the retinal nerve fiber layer, macula, and retinal layer thicknesses in children. Am J Ophthalmol. 2013;155(2):354-60.

13. Ferreira BG, Marinho DR, Diligenti FT. Effects of sub-Tenon's triamcinolone injections in patients with uveitis. Arq Bras Oftalmol. 2018;81(4):323-9.
14. Angeles-Han ST, Lo MS, Henderson LA, et al. Childhood arthritis and rheumatology research alliance consensus treatment plans for juvenile idiopathic arthritis-associated and idiopathic chronic anterior uveitis. Arthritis Care Res (Hoboken). 2019;71(4):482-91.

15. Gautam Seth N, Kaur S, Yangzes S, et al. Ophthalmic complications in pediatric uveitis. Ocul Immunol Inflamm. 2020;2:1-6.

16. Sancho L, Kramer M, Koriat A, Eiger-Moscovich M, Sharon Y, Amer R. Complications in intermediate uveitis: prevalence, time of onset, and effects on vision in short-term and long-term follow-up. Ocul Immunol Inflamm. 2019;27(3):447-55.

17. Gautam Seth N, Yangzes S, Thattaruthody F, et al. Glaucoma secondary to uveitis in children in a tertiary care referral center. Ocul Immunol Inflamm. 2019;27(3):456-64.

18. Eiger-Moscovich $\mathrm{M}$, Tomkins-Netzer $\mathrm{O}$, Amer $\mathrm{R}$, et al. Visual and clinical outcome of macular edema complicating pediatric noninfectious uveitis. Am J Ophthalmol. 2019;202:72-8.

19. Kothari S, Foster CS, Pistilli M, et al. The risk of intraocular pressure elevation in pediatric noninfectious uveitis. Ophthalmology. 2015;122(10): 1987-2001. 